\title{
Internet of Things: A Secure Cloud-Based MANET Mobility Model
}

\author{
Tanweer Alam \\ Computer Science Department, Islamic University of Madinah, Madinah, Saudi Arabia \\ E-mail: tanweer03@iu.edu.sa
}

Please cite this article as follows.

Tanweer Alam. " Internet of Things: A Secure Cloud-Based MANET Mobility Model.", International Journal of Network Security. Vol 22(3), 2020. DOI: 10.6633/IJNS.202005_22(3).17

\begin{abstract}
Connected devices such as Smart Home Automation Gateway, Smart Air Conditioners, Smart Hubs, Smart Thermostat, Color Changing Smart Lights, Smart Mobile phones, Smart Watches and Smart Tablets, etc. are omnipresent in our everyday lives and are becoming a valuable tool with wireless networking features using different wireless protocols commonly used. Access points allow interactions between users within an Internet of Things ecosystem infrastructure. These smart devices are automatically connected, and a network is formed by themselves. However, there are many challenges throughout this established network of its own for secure communication. Security has been perceived as a popular barrier to adopting the cloud model of internet realism. The storage and resource management may be in the cloud environment is a distributed structure that places the world in a raised situation with many concerns over its weaknesses, security risks and difficulties. Different participation parties have broadened those issues depending on the viewpoint and goal of each party. The author primarily addresses the causes of challenges and difficulties related to security, reliability, privacy and availability of services from the Cloud point of view. Connectivity Security has been identified as one of cloud computing's most critical issues where resolving such an issue would result in constant growth in the use and popularity of cloud computation. The purpose of this study is to build a mobile ad hoc network mobility model framework using cloud computing to provide secure Internet of Things communication between smart devices. The major contribution relates a new methodology to ensure secure communication with the 5G network of smart devices using the internet. The approach uses a desired study's accurate and effective simulation and can be applied in an Internet of Things structure. This research would create a new connectivity architecture to address the problem of secure communication between smart devices in $5 \mathrm{G}$ networking.
\end{abstract}

Keywords-Mobile Ad-Hoc Networks (MANET); Mobility Models; Cloud Computing; Internet of Things; Smart Devices; 5G Heterogeneous Network;

\section{INTRODUCTION}

This research is a move forward into the field of cloud computing and the Internet of Things in $5 \mathrm{G}$ heterogeneous networks as the author suggested a framework for the mobility model using cloud computing to connect smart devices together on the internet. In this study, the proposed research work is an improvement and implementation of current mobile ad-hoc network communication using the cloud within the internet of things environment. This research provides an approach that is able to develop a new framework for the secure communication of smart devices on the internet. This research was used in the right and efficient simulation of the targeted research and can be applied within the IoT framework. Today's wireless network is composed of cells in a specific area within its range. Every cell includes a base station, which can be linked through wired or wireless networks [1]. Nowadays, smart devices provide very useful 
Wi-Fi Direct functionality [2]. By using this technology any device can communicate and create a MANET network with neighborhood devices [3], [4]. When one device has internet then the same device can connect to the cloud and build a MANET of the smart device [5]. The growth of the internet of smart devices is estimated to increasingly connect with 50 billion smart devices by 2020 [6]. Such growth would not rely on the population of humankind, however on the fact that we regularly use smart devices [7].

A reality of objects that are connected is that they can communicate between the device to the device [8]. These devices are going to talk to one another [9]. However, one of the most comprehensive issues is the monitoring and tracking of movable devices [10]. A concept of the internet of things could be represented as "a ubiquitous and pervasive network that further facilitates the monitoring of physical devices and control through gathering, processing, and also analyzing the information using sensor network" [11], [12]. The evolutionary paradigm allows the users to deliver effortless access to a network of computing resources, where users can easily scale up or down their expec tations with the service provider's irrelevant interactions [13].

Started from 2008, the internet of things is growing exponentially to connect the physical objects using the internet. These physical objects are connected to an intelligent database that has smart data storage [14]. To recognize the physical object, houses, people's image, location etc. the framework uses image recognition techniques. The internet of things is now moving from information technology to operational technology, i.e. IPV4 (man 2 machines) to IPV6 (machines 2 machines) and It integrates sensors, smart devices and such Smart Grid interfaces [15]. Throughout a general sense, each user has questions about flaws in cloud computing and challenges that might keep them from achieving their goals. The three innovations that have contributed to growth on the internet of things. A ubiquitous computation that has the intelligent capability of physical objects operating on the computation platform [16]. The Internet Protocol (IPV6) uses universal computing covering the network area and enabling machine-to-machine communication. The IPv4 internet has a weakness of connecting billions of smart devices together, but IPv6 internet is possible because it enables the internet of things to link billions of smart devices together [17]. Communication in ubiquitous computing with the use of sensor connectivity in fixed cell networks or mobility should be periodically improved to allow the advancement of smart devices internet including multi-sensor systems to store, compute, analyze and process capabilities that require smaller size and lowest energy [18]. This article shows the main contribution that links a new secure model of communication using cloud computing and MANET technologies in the Internet of Things environment [19]. A secured data self-destructing scheme in Cloud computing is presented in the article [34].

The idea of communication security depends on three main points in the architectural design of the internet of things. 1. Managing information receiving from millions of sensors in a centralized smart device collection system is not easy. 2. Managing network resources in a large network that can gather environment information from the centralized framework is not an easy task. 3. Managing sensors which execute the same kind of data-parallel and stored in the centralized system is very complicated [20]. 


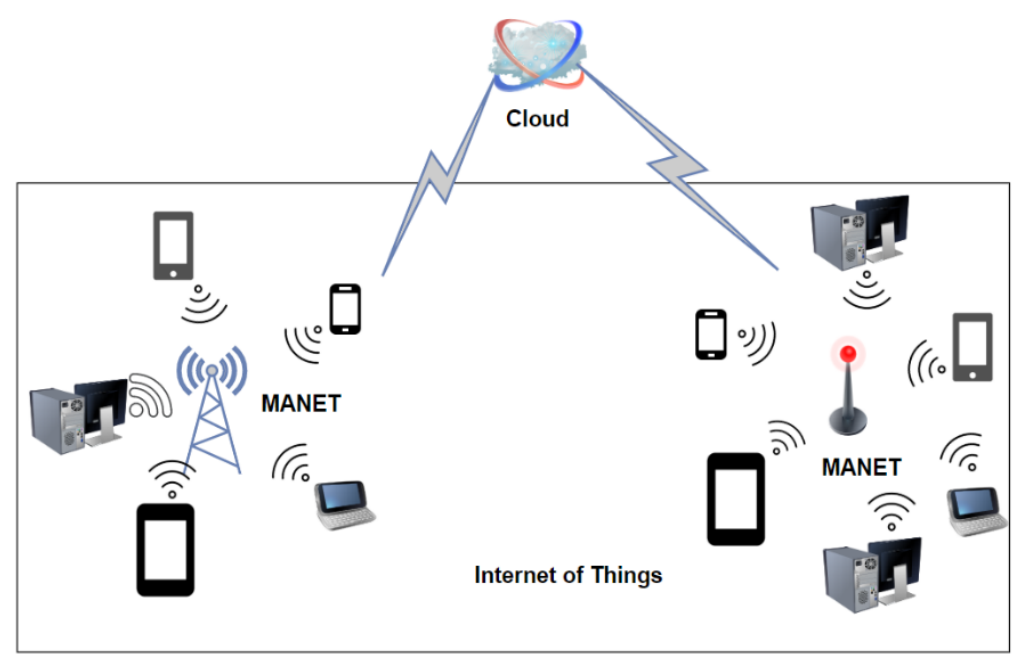

Figure 1. Internet of Things with Cloud and MANET

Cloud computing would have been one of the most famous concepts in computation [21]. This also comes out of recent computing paradigms advances that combine parallel computing, grid computing, disseminated computing, and other computing methodologies. The cloud computing provides its users with three basic models of administration: SaaS, PaaS, and IaaS. The first one is SaaS, Software as a service (SaaS) is intended primarily for end-users who need to use the software as part of their everyday lives. Secondly, Platform as a service (PaaS) is primarily intended for software developers who need technologies to develop their software as well as implementation. The principal aim of Infrastructure as a Service (IaaS) is to network architects who need infrastructure functionality [22]. A more essential element of the framework is the communication security challenges and threats to communicate smart device's internet from a cloud point of view. The cloud environment offers the power of shared resources to its end users. The cloud providers employ multi-tenancy to eventuate the concept of exchanging. Basically, the implementation of a maximum throughput of recourses is provided as software architecture.

MANET is a very successful system to always get connected at any time anywhere. The cloud computing provides data storage and access service. Cloud and MANET integration provides the services for cloud access within MANET of devices connected. The smart devices are able to connect to each other in the area where there are no network facilities [23]. Among smart devices, the MANET can be formed automatically. And it can use the cloud service if one device has the internet in the group of smart devices. MANET mobility model implementation in the smart device to smart device communication can be very efficient and useful to save energy as well as increase the efficiency in the Internet of Things. This approach of cloud-based services in the MANET model for the device to device communication can be a very useful approach to enhance the capabilities of smart devices in the internet of things environment. MANET connected devices are also able to use cloud service to discover neighborhood devices and exchange information. The proposed approach includes MANET and cloud computing on the internet of smart devices that can be useful in the $5 \mathrm{G}$ heterogeneous network.

The rest of the paper is organized as follows: Section 2 shows the literature survey, section 3 presents the methodologies, section 4 presents the Cloud-MANET Mobility Model and section 5 represents the conclusion of the research.

\section{Literature Survey}

In 2012, Lacuesta et. Al. was published an article [24] on the internet of things trust. The smart devices that can take part in the MANET networks can be quite different from one another. The Network can be embedded with sensors, mobile apps, home appliances, or other types of devices, will need to work together 
to increase and enhance customer satisfaction. Some of these tools may have limited resources to run, sometimes even non-existent resource constraints, they must work to optimize network traffic. The authors were focusing attention on spontaneous networks in this article. They were proposed a secure ad hoc spontaneous network, based on direct peer-to-peer interaction in the Internet of Things [24]. A secured and authenticated anonymous data access on cloud in the MANET network is presented in the article [32]. In the article [33], the zone Based MANET Routing Protocol with a Genetic algorithm is presented.

In 2015, Airehrour, D.et. al. was published an article [25], they were developed various secure routing protocols for MANETs which could be used to establish secure routing protocols for the Internet of Things, so the analysis of these secure MANET routing protocols will provide a roadmap for the development and implementation of security in the Internet of Things and Cloud Computing. In this paper, the authors also provide secure routing protocols in MANETs while offering some secure routing features for IoT routing to ensure confidentiality and integrity. They also discussed research trends and future directions in the field of IoT network security [25].

In 2017, Al-Zaidi, R. et. Al. was published an article [26], the authors were presented the Internet of Things (IoT) technology over Ship Ad-hoc Networks (SANET) as a maritime data acquisition and cartography system The Ships were recommended to communicate over a Very High Frequency (VHF) which is already available on most ships and are fitted with multiple sensors such as sea level, temperature, wind speed and direction and so on. $5 \mathrm{G}$ base station nodes onshore server sinks for the data collected and are fitted with data aggregation and processing capabilities for Mobile Edge Computing. Finally, the sensory data is aggregated on the internet in a central repository to generate up-to-date digital cartography solutions.

In 2018, Tanweer Alam and Mohamed Benaida have published an article [27], they were proposed a framework that can access and deliver cloud services to the MANET users through their smart devices in the IoT. Also, the proposed framework was performed where all computations, data handling, and resource management. MANETs can connect to the cloud and can use cloud services. The main contribution in this research links a new methodology for providing secure communication on the internet of smart devices using MANET. In this research, the methodology uses the correct and efficient simulation of the desired study and can be implemented in a framework of the Internet of Things in the future.

In 2020, H.Riasudheen, et. Al. are published an article [28], the Device-to-Device (D2D) interaction in 5G networks has increased the number of devices and the rate of data transmission in Cloud Assisted-Mobile Ad Hoc Networks of smart devices. Furthermore, due to mobility, connection failure overload networking and limited battery energy, the connection between the smart devices has to be regularly renewed. It consumes lots of energy during this time in searching for and connecting the smart devices. Compared with other existing network mobility models and routing protocols, this proposed research provides better performance.

\section{Methodologies}

Discovering the smart devices in MANET, the hidden Markov model is utilized in the 2Dimensional plane zone. The framework is connected in this area and smart devices can move inside this area and search another smart devices. The transition matrix is formed in the area of MANET for discovering all the smart devices. Some parameters are used for discovering smart devices as follows.

Suppose, $S=S_{1}, S_{2} \ldots \ldots . S_{N}$ where $S$ is the state, $S_{1}$ is the first state, $S_{2}$ is the second state, etc. Every cell depends on one state in $\mathrm{S}$.

Transition matrix probability $\mathrm{P}=\mathrm{P}_{\mathrm{ij}}(1 \leq \mathrm{i} \leq \mathrm{N})$ where $\mathrm{P}_{\mathrm{ij}}$ characterized to move likelihood from $\mathrm{S}_{\mathrm{i}}$ to $\mathrm{S}_{\mathrm{j}}$ in the Transaction Matrix. 
The probability $\mathrm{P}_{\mathrm{ij}}$ is just significant if $\mathrm{Si}, \mathrm{S}_{\mathrm{j}}$ is neighborhood states in the $2 \mathrm{D}$ plane. The states will rearrange to move up, down, left and right in the 2D plane [29]. The left components within the framework are all 0s initially. The hidden Markov model is represented in the following transition matrix.

The smart device in each cell represented by $\Pi=\prod_{i}(1 \leq \mathrm{i} \leq \mathrm{N})$.

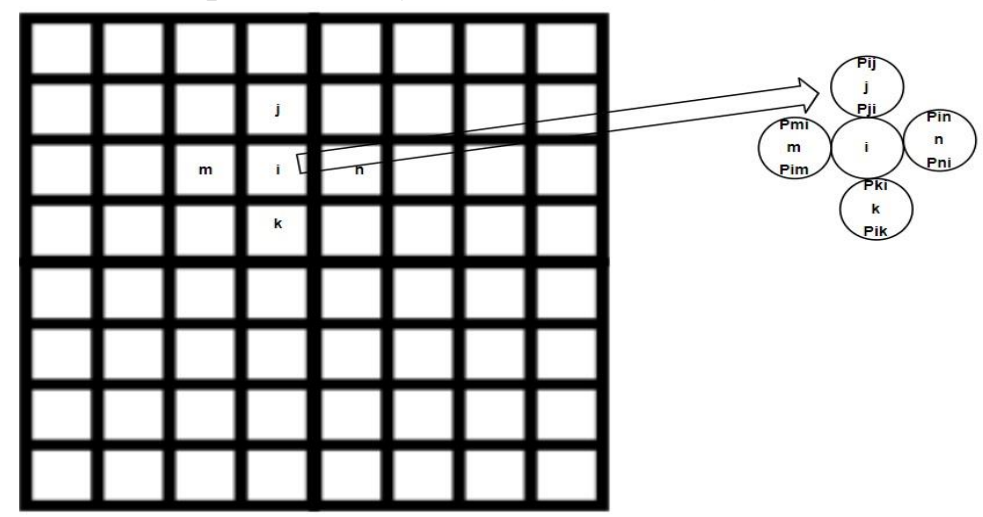

Figure 2: HMM model transition matrix representation

Smart devices in MANET can be used to discover the signals using the Viterbi algorithm in the 2D plane. Suppose $\mathrm{O}_{1}, \mathrm{O}_{2}, \ldots, \mathrm{O}_{\mathrm{n}}$ are the observation of discovering the devices in the 2D plane. Each smart device sends a report of observations during a time period [30]. The algorithm discovers the way at each step by maximizing the throughput of the smart devices. The process is too much time consuming for the rush of devices in the specific zone. This whole process can be accomplished by essentially joining the sub-track at every cell of the area. The fact is that the HMM model has been utilized as a part of the target to discover the device connected to the appropriate MANET of smart devices [31]. Hidden Markov model depends on the state's probability and the transition matrix represents the information in every cell of the area. If a smart device enters a new cell then it removes previous data and updates the information.

Discovering the smart devices, the gradient model works to find the devices and share the information among the smart devices. Eventually, when a smart device recognizes another smart device, the gradient value will set 1 also discover another smart device in the area where MANET is formed.

Apply the physical law and find the distance between the two smart devices that is proportional to one upon the distance of the event.

Device (distance) $\alpha 1 /$ event ${ }^{\text {distance }}$

Gradient over time is represented by the following formula.

The gradient with respect to time $\mathrm{t}$ as follows:

Gradient (t) $\alpha \quad\left\{\begin{array}{cr}1 & t=0 \\ \text { event }^{-t} & 0<\mathrm{t}<\text { Totaltime } \\ 0 & \mathrm{t}>=\text { Totaltime }\end{array}\right.$

The Gradient model finds the gradient distribution over the time period. When $\mathrm{t}=0$ then the gradient value will be 1 and if time is greater than the total time then the value of gradient will be 0 , otherwise, the gradient is proportional to one upon time power of the event $\left(\right.$ event $\left.^{-t}\right)$. 


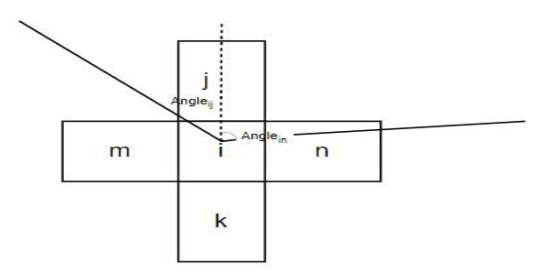

Figure 3: Motion in Gradient-based Model

Smart devices are held in the range of MANET that considers coverage and connectivity of the Wi-Fi ad hoc network. Each smart device is expected to have a settled Wi-Fi area. Firstly, the wi-fi Ad Hoc Network is dynamically connected. The MANET is inactive all the time, when one device wants to make the connection with another device then it creates a connection with their neighborhood device. The inactive smart device additionally occasionally awakens to enter into an active device. The active state detects the devices and connects dynamically.

\section{Cloud-MANET Mobility Model}

Device-to-device connectivity will improve the overall performance, expand coverage and minimize the energy consumption of mobile communications through communicating directly. Smart device to smart device communication in the cloud-MANET framework of the internet of things is a novel methodology that discovers and connected nearby smart devices without a centralized system. In the existing cellular network, not allowed to connect all smart devices without centralized infrastructure even if they are very near. This proposed technique would be very useful in the machine to machine (M2M) communication because, in the M2M network, there are many devices nearby. Therefore, the implementation of the MANET model in the smart device to smart device communication can be very efficient and useful to save power as well as efficiency. Cloud-based MANET mobility model for the device to device communication can be a very useful approach to enhance the capabilities of smart devices in 5G.

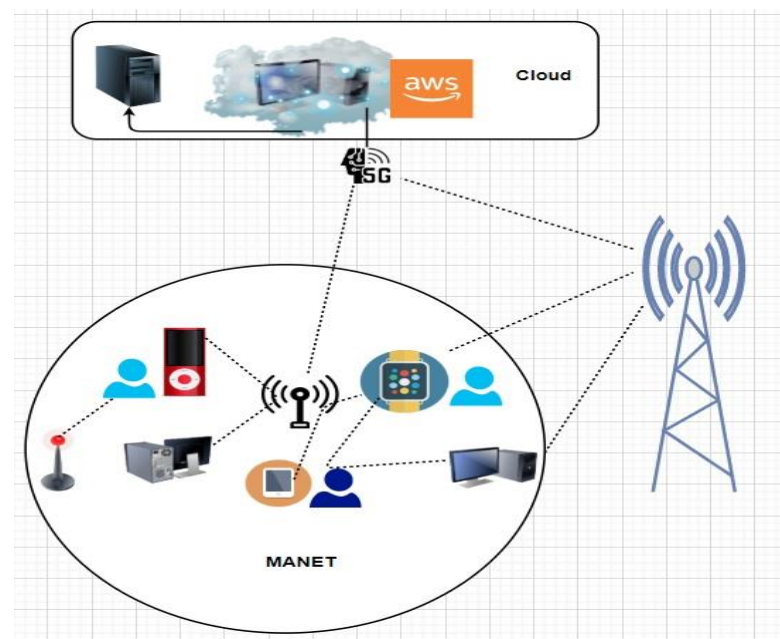

Figure 4: Cloud-MANET mobility model

When two or more smart devices wish to communicate in the Cloud-MANET model on the internet of things then communication security is the main challenge. Throughout the Cloud-MANET model, the smart devices are dynamically joined and created a network on their own. Also, they can access the cloud service. But there are more challenges for secure communication in this own created network that access cloud services. Cloud-MANET is a kind of wireless network that is self-organizing and auto connected in a decentralized system and accessed the cloud. Each device in MANET can be moved freely from one location 
to another in any direction within the range of Wi-Fi. Several MANETs can connect with the same cloud and they can use cloud services. MANET model of smart devices in local communication can work very well using the cloud, it is failed when it connects in exist wired networks. Every smart device needs to search for neighborhood devices. A most important question arises here: How will communication secure in the public cloud and MANET? The answer is yes, it is possible through the cloud-MANET model that is implemented and integrated with mobile apps and tested. Cloud MANET mobility model is an integrated model of Cloud computing and MANET networks. MANET model is depended on the mobility of its nodes and connectivity, resources such as storage and power consumption. Cloud providers retain network infrastructure, storage facilities, and software applications that support flexibility, efficiency, and ability. The Cloud-MANET mobility model allows the smart devices to communicate with another smart device, However, at least one smart device must be connected to cellular or Wi-Fi networks and access the internet. Every smart device of MANET should be registered in the cloud. If a MANET is activated then cloud services will activate in real-time and provide services to the smart devices of MANET to communicate. Smart devices send a request to the cloud to provide a session of connection. The connection can be described as the probabilistic function as follows.

Session $($ life $))=\left(\frac{\int_{\text {life }}^{\infty}\left(\left(\frac{1}{2}\right)-\left(\frac{1}{2}\right) \operatorname{erf}\left(\log \left(\frac{u}{\mu}\right) \div \sqrt{2} \sigma\right)\right) d u}{\left(\frac{1}{2}\right)-\left(\frac{1}{2}\right) \operatorname{erf}\left(\log \left(\frac{i f e}{\mu}\right) \div \sqrt{2} \sigma\right)}\right)$

The integral limit is 0 tends to $\infty$ in the session life. The above probabilistic method requires to compute the values of $\sigma$ and $\mu$ for every smart device when they have a session with the cloud.

$\sigma$ and $\mu$ are two parameters that are related to the connection establishment among MANETs and Cloud service. It can be measured through smart devices using the following method.

$\mathrm{e}^{\mu(1 / 2) \sigma 2}$.

If a smart device estimates the connection life between MANET and Cloud then it will transfer or receive secured data.

Connection will be active and can connect. The author is considered that every smart device is assured to establish the route between MANET and cloud when they create a session. Smart devices can move through the maximum speed from one location to another location by using the Gauss-Markov mobility framework. Calculate the moving speed and direction of the smart device within the MANET range by using the following formula.

Speed $^{t}=\lambda$ Speed $_{t-1}+(1-\lambda)$ Speed $_{+} \sqrt{ }\left(1-\lambda^{2}\right)$ Speed $_{t-1}^{G}$

and

Direction $_{\mathrm{s}}=\lambda$ Direction $_{\mathrm{t}-1}+(1-\lambda)$ Direction $^{-} \quad+\sqrt{\left(1-\lambda^{2}\right) \text { Direction }_{t-1}^{G}}$

$\lambda$ is used as a random degree when computing speed as well as the direction of smart device in time $(t)$.

Transmission $\left(\mathrm{t}_{\mathrm{s}}\right)$ of information $\left(\mathrm{I}_{\mathrm{k}}\right)$ among the number of smart devices $\left(\mathrm{S}_{\mathrm{n}}\right)$ can be estimated during the time interval $\left[\mathrm{t}_{\mathrm{i}}, \mathrm{t}_{\mathrm{i}-1}\right]$. Smart devices can move within the MANET and access the cloud service using the multidimensional function $\left(\varepsilon^{\mathrm{k}}\right)$.

$\varepsilon \mathrm{k}=\mathrm{CSn} \times \mathrm{tk} \times \mathrm{Ik}$

where $\mathrm{k}=0,1,2,3 \ldots \ldots \infty(+\mathrm{ve})$.

When smart devices have moved outside the MANET then $\mathrm{k}$ will be negative. We have considered that the transformation of information simultaneously happens. The probability is proportional to the one divide by information $\left(\mathrm{I}_{\mathrm{k}}\right)$.

$\mathrm{P}^{k} \propto \frac{1}{I_{k}}$

Probability density function for transmission is calculated mathematically as. 
$\mathrm{P}_{\mathrm{k}}\left(\mathrm{S}_{\mathrm{n}} \mid \varepsilon_{\mathrm{k}}, \mathrm{I}_{\mathrm{k}}, \mathrm{t}_{\mathrm{k}}\right)=\quad 1 \quad-\int_{-\infty}^{\infty} M_{Q}\left(\sqrt{\frac{2 \gamma^{2}}{1-\gamma^{2}} \times \frac{S_{n}}{t_{k}}}, \sqrt{\frac{S_{n}}{1-\gamma^{2}} \times 2 \gamma}\right)$

Discover the smart device and find the new position of the smart devices in MANET using the following algorithm.

Find the position $\left(\mathrm{X}_{1}, \mathrm{Y}_{1}\right)$ of the smart device in the MANET.

Find the current Speed (s) of the moving device in MANET.

Speed can get using the following formula.

Speed $(\mathrm{s})=$ distance $(\mathrm{d}) /$ time $(\mathrm{t})$.

Whether time $=\mathrm{t}$ and angle is $\theta$ (positive) then we consider the new location of the smart device using the following formula.

$\mathrm{X}_{2}=\mathrm{X}_{1}+\mathrm{s} * \mathrm{t} * \cos (\theta)$

$\mathrm{Y}_{2}=\mathrm{Y}_{1}+\mathrm{s} * \mathrm{t} * \sin (\theta)$

When $\theta$ is negative then

$\mathrm{X}_{2}=\mathrm{X}_{1}-\mathrm{s} * \mathrm{t} * \cos (\theta)$

$\mathrm{Y}_{2}=\mathrm{Y}_{1}-\mathrm{s} * \mathrm{t} * \sin (\theta)$

4. Obtain the real Location of smart device.

Location Loc=get_New Location (new Point(smart device Location)); Example: Loc $=\left(\mathrm{x}_{1}, \mathrm{y}_{1}\right)$

5. Obtain the theoretical location

Location ref=get NewRef Location (new Point(smart device Location)); Example: ref=( $\left.\mathrm{x}_{2}, \mathrm{y}_{2}\right)$

6. Obtain the distance $\mathrm{d}$ between Loc and ref.

$\mathrm{d}=\sqrt{ }\left(x_{2}-x_{1}\right)^{2}-\left(y_{2}-y_{1}^{2}\right)$

obtain the random location $(\mathrm{X}, \mathrm{Y})$ of smart device at the diagonal of triangle can find using the following formula.

$\mathrm{X}=$ Math.random $(\operatorname{d} \cdot \operatorname{get} \mathrm{X}())$;

$\mathrm{Y}=$ Math.random(d.getY());

Obtain the actual location of the smart device according to the diagonal of the triangle, it may be on the diagonal or upper or lower than the diagonal. when the device is upper than the diagonal then increase the value of $\mathrm{X}$ and $\mathrm{Y}$.

$\mathrm{X}=\mathrm{X}+\delta \mathrm{X}$

$\mathrm{Y}=\mathrm{Y}+\delta \mathrm{Y}$;

else

$\mathrm{X}=\mathrm{X}-\delta \mathrm{X}$

$\mathrm{Y}=\mathrm{Y}-\delta \mathrm{Y}$

9. Return new Location(X, Y).

This Cloud-MANET mobility model had been implemented and tested. The MANET is building and verified on three Samsung mobile phones. The Amazon Web Services (AWS) are used for implementing cloud services with MANET. The amazon cloud service will connect to the MANETs and provide a session for connection. At least one device should be registered in the cloud and access the cloud and share it in MANET.

\section{CONCLUSION}

Cloud-MANET mobility model can play a vital role in 5G. It can enhance the efficiency and speed of communication in the cloud and MANET. The cloud paradigm is based on a distributed architecture, it is inherited some risks and vulnerabilities that are related to distributed computing. Communication security threats and challenges that rely on behind the lure of cloud computation. Cloud-MANET mobility model has been developed and tested. One device start service of MANET as well as connected with the cloud and start 
to share connection and exchange information. The author has used cloud service from Amazon cloud. This study showed successfully and expectation for a future scope in this field. The author reached the conclusion that this kind of network could help people in many situations, some of them in critical situations after researching a lot of how MANET networks work and which are its advantages and disadvantages.

\section{REFERENCES}

[1] Brownrigg, Edwin B., and Thomas W. Wilson. "Wireless network system and method for providing same." U.S. Patent 6,044,062, issued March 28, 2000.

[2] Abu-Sharkh, Osama MF, Esam AlQaralleh, and Omar M. Hasan. "Adaptive device-to-device communication using Wi-Fi Direct in smart cities." Wireless Networks 23, no. 7 (2017): 2197-2213.

[3] Alam, Tanweer. "Middleware Implementation in Cloud-MANET Mobility Model for Internet of Smart Devices", International Journal of Computer Science and Network Security, 17(5), 2017. Pp. 86-94

[4] Alam T, Benaida M. CICS: Cloud-Internet Communication Security Framework for the Internet of Smart Devices. International Journal of Interactive Mobile Technologies (iJIM). 2018 Nov 1;12(6):74-84. DOI: https://doi.org/10.3991/ijim.v12i6.6776

[5] Tanweer Alam, Baha Rababah, "Convergence of MANET in Communication among Smart Devices in IoT", International Journal of Wireless and Microwave Technologies(IJWMT), Vol.9, No.2, pp. 1-10, 2019. DOI: 10.5815/ijwmt.2019.02.01

[6] Tanweer Alam, "IoT-Fog: A Communication Framework using Blockchain in the Internet of Things", International Journal of Recent Technology and Engineering (IJRTE), Volume-7, Issue-6, 2019.

[7] Tanweer Alam, "Blockchain and its Role in the Internet of Things (IoT)", International Journal of Scientific Research in Computer Science, Engineering and Information Technology, vol. 5(1), pp. 151-157, 2019. DOI: https://doi.org/10.32628/CSEIT195137

[8] Alam, Tanweer. (2018) "A reliable framework for communication in internet of smart devices using IEEE 802.15.4." ARPN Journal of Engineering and Applied Sciences 13(10), 3378-3387.

[9] Tanweer Alam, "A Reliable Communication Framework and Its Use in Internet of Things (IoT)", International Journal of Scientific Research in Computer Science, Engineering and Information Technology (IJSRCSEIT), Volume 3, Issue 5, pp.450-456, May-June.2018 URL: http://ijsrcseit.com/CSEIT1835111.

[10] Alam, Tanweer, and Mohammed Aljohani. "Design and implementation of an Ad Hoc Network among Android smart devices." In Green Computing and Internet of Things (ICGCIoT), 2015 International Conference on, pp. 1322-1327. IEEE, 2015. DOI: https://doi.org/10.1109/ICGCIoT.2015.7380671

[11] Alam, Tanweer, and Mohammed Aljohani. "An approach to secure communication in mobile adhoc networks of Android devices." In 2015 International Conference on Intelligent Informatics and Biomedical Sciences (ICIIBMS), pp. 371-375. IEEE, 2015. DOI: https://doi.org/10.1109/iciibms.2015.7439466

[12] Aljohani, Mohammed, and Tanweer Alam. "An algorithm for accessing traffic database using wireless technologies." In Computational Intelligence and Computing Research (ICCIC), 2015 IEEE International Conference on, pp. 1-4. IEEE, 2015. DOI: https://doi.org/10.1109/iccic.2015.7435818

[13] Alam, Tanweer, and Mohammed Aljohani. "Design a new middleware for communication in ad hoc network of android smart devices." In Proceedings of the Second International Conference on Information and Communication Technology for Competitive Strategies, p. 38. ACM, 2016. DOI: https://doi.org/10.1145/2905055.2905244

[14] Alam, Tanweer. "Fuzzy control based mobility framework for evaluating mobility models in MANET of smart devices." ARPN Journal of Engineering and Applied Sciences 12, no. 15 (2017): 4526-4538. 
[15] Alam, Tanweer, Arun Pratap Srivastava, Sandeep Gupta, and Raj Gaurang Tiwari. "Scanning the Node Using Modified Column Mobility Model." Computer Vision and Information Technology: Advances and Applications 455 (2010).

[16] Alam, Tanweer, Parveen Kumar, and Prabhakar Singh. "SEARCHING MOBILE NODES USING MODIFIED COLUMN MOBILITY MODEL.", International Journal of Computer Science and Mobile Computing, (2014).

[17] Alam, Tanweer, and B. K. Sharma. "A New Optimistic Mobility Model for Mobile Ad Hoc Networks." International Journal of Computer Applications 8.3 (2010): 1-4. DOI: https://doi.org/10.5120/1196-1687

[18] Singh, Parbhakar, Parveen Kumar, and Tanweer Alam. "Generating Different Mobility Scenarios in Ad Hoc Networks.", International Journal of Electronics Communication and Computer Technology, 4(2), 2014

[19] Sharma, Abhilash, Tanweer Alam, and Dimpi Srivastava. "Ad Hoc Network Architecture Based on Mobile Ipv6 Development." Advances in Computer Vision and Information Technology (2008): 224.

[20] Aljohani, Mohammed, and Tanweer Alam. "Real Time Face Detection in Ad Hoc Network of Android Smart Devices", Advances in Computational Intelligence: Proceed-ings of International Conference on Computational Intelligence 2015. Springer Singa-pore, 2017.DOI: https://doi.org/10.1007/978-981-10-2525-9_24

[21] M. Aljohani and T. Alam, "Design an M-learning framework for smart learning in ad hoc network of Android devices," 2015 IEEE International Conference on Computational Intelligence and Computing Research (ICCIC), Madurai, 2015, pp. 1- 5. DOI: https://doi.org/10.1109/ICCIC.2015.7435817

[22] Alam, Tanweer. "Tactile Internet and its Contribution in the Development of Smart Cities." arXiv preprint arXiv:1906.08554 (2019).

[23] Tanweer Alam, "5G-Enabled Tactile Internet for smart cities: vision, recent developments, and challenges", JURNAL INFORMATIKA, Vol. 13, No 2, July 2019, pp. 1-10, DOI: 10.26555/jifo.v13i2.a13426

[24] Lacuesta, R., Palacios-Navarro, G., Cetina, C., Peñalver, L. and Lloret, J., 2012. Internet of things: where to be is to trust. EURASIP Journal on Wireless Communications and Networking, 2012(1), p.203.

[25] Airehrour, D. and Gutierrez, J., 2015. An analysis of secure MANET routing features to maintain confidentiality and integrity in IoT routing. International Conference on Information Resources Management (CONF-IRM) at AIS Electronic Library (AISeL).

[26] Al-Zaidi, R., Woods, J., Al-Khalidi, M., Alheeti, K.M.A. and McDonald-Maier, K., 2017, May. Next generation marine data networks in an IoT environment. In 2017 Second International Conference on Fog and Mobile Edge Computing (FMEC) (pp. 50-55). IEEE.

[27] Alam, T. and Benaida, M., 2018. The Role of Cloud-MANET Framework in the Internet of Things (IoT). International Journal of Online and Biomedical Engineering (iJOE), 14(12), pp.97-111.

[28] Riasudheen, H., Selvamani, K., Mukherjee, S. and Divyasree, I.R., 2020. An efficient energy-aware routing scheme for cloud-assisted MANETs in 5G. Ad Hoc Networks, 97, p.102021.

[29] Tanweer Alam and Mohammed Aljohani, "Decision Support System for Real-Time People Counting in a Crowded Environment", I.J. of Electronics and Information Engineering, Vol.12, No.1, PP.34-41, Mar. 2020.

[30] T. Alam, "Cloud Computing and its role in the Information Technology", IAIC Transactions on Sustainable Digital Innovation (ITSDI), vol. 1, no. 2, pp. 108-115, Feb. 2020.

[31] Alam, Tanweer, Abdulrahman A. Salem, Ahmad O. Alsharif, and Abdulaziz M. Alhejaili. "Smart Home Automation Towards the Development of Smart Cities." APTIKOM Journal on Computer Science and Information Technologies 5, no. 1 (2020). 
[32] Bardiya, M.P.D. and Ramteke, P., Secured and Authenticated Anonymous Data Access on Cloud in MANET. International Journal of Advanced Research in Computer and Communication Engineering, ISSN, 2278(1021), pp.128-137, 2015.

[33] Preetha, V. and Chitra, K., 2018. ZBMRP: Zone Based MANET Routing Protocol with Genetic Algorithm and Security Enhancement Using Neural Network Learning. IJ Network Security, 20(6), pp.1115-1124.

[34] Liu, L., Li, Y., Cao, Z. and Chen, Z., 2020. A Note On One Secure Data Self-Destructing Scheme in Cloud Computing. International Journal of Network Security, 22(1), pp.36-40. 